\title{
Childhood adversities, history of mental disorders, anxiety-depressive symptoms are associated with dual-harm in violent offenders
}

\section{Xiaoping Wang ( $\sim$ xiaop6@csu.edu.cn )}

Central South University, National Technology Institute on Mental Disorders

\section{Ying Huang}

Central South University, National Technology Institute on Mental Disorders

Simei zhang

Shenzhen Kangning Hospital

Shaoling Zhong

The Affiliated Brain Hospital of Guangzhou Medical University

Ningzhi Gou

Central South University, National Technology Institute on Mental Disorders

Qiaoling Sun

Central South University, National Technology Institute on Mental Disorders

Huijuan Guo

Central South University, National Technology Institute on Mental Disorders

Ruoheng Lin

Central South University, National Technology Institute on Mental Disorders

Weilong Guo

Central South University, National Technology Institute on Mental Disorders Hui Chen

Central South University, National Technology Institute on Mental Disorders Jizhi Wang

Central South University, National Technology Institute on Mental Disorders Jiansong Zhou

Central South University, National Technology Institute on Mental Disorders

\section{Research Article}

Keywords: Dual-harm, childhood adversities, mood disorder, violent offender, China

Posted Date: February 21st, 2022

DOl: https://doi.org/10.21203/rs.3.rs-1316774/v1 
License: (c) (i) This work is licensed under a Creative Commons Attribution 4.0 International License. Read Full License 


\section{Abstract}

Backgroud: The coexistence of self-harm and aggression, which is referred to as dual-harm, is commonly seen in forensic population. However, self-harm and aggression have often been studied separately, and the factors associated with dual-harm remain unclear. This study aimed to explore potential risk factors associated with co-occurring self-harm among individuals with violent behaviors.

Methods: This multi-center case-control study was conducted from May 2013 to January 2016 and involved seven qualified forensic institutes located in seven provinces in China. Participants were individuals with violent behaviors who were suspected to have mental disorders. Lifetime history of selfharm was obtained by a self-report questionnaire, and violent behaviors were assessed with the use of participants' forensic archive. Sociodemographic and clinical information were collected using a selfdesigned standardized data collection form, and childhood adverse experience was assessed using a clinician-rated scale designed by our research team. The psychopathy checklist- Revised was used to assess psychopathic traits and the Brief Psychiatric Rating Scale was used to assess psychiatric symptoms of the participants.

Results: A total of 429 participants were enrolled in the current study. Of them, 74 (17.2\%) with self-harm history assigned into the dual-harm group (D-H) and 355 (82.8\%) without self-harm history assigned into the violence-only group ( $\mathrm{V}-\mathrm{O})$. According to the binary logistic regression analysis, current diagnosis of mood disorder (OR=3.03, 95\% Cl: 1.15 - 7.97), child abuse (OR=2.92, 95\% Cl: 1.33 - 6.40), parental death $(\mathrm{OR}=2.79,95 \% \mathrm{Cl}: 1.14-6.81)$, and the score of the affective subscale in $B P R S(\mathrm{OR}=1.77,95 \% \mathrm{Cl}: 1.31$ 2.39) were significantly associated with dual-harm.

Conclusions: Our study suggested the necessity of integrated evaluation of self-harm among violent offenders. Childhood adverse experiences and psychiatric symptoms in this population require special attention.

\section{Background}

Mounting evidence suggested that some individuals engage in both self-harm and aggression during their lifetime[1-3]. The co-existence of self-harm and aggression is termed as dual-harm[4]. A large population-based investigation reported that the prevalence of self-harm co-occurring with violent crime was approximately $0.4 \%[1]$. There is an increasing risk of unnatural death and development of riskier patterns of harmful behaviors among dual-harm individuals. In 2019, a study reported that the risk of unnatural death in dual harmers was significantly higher than in the general population or those engaging in violence towards others or self only[5]. Compared to those who engage in only self-harm or other-harm, individuals with dual-harm were likely to be different both in quality (e.g., methods of harm) and quantity (e.g., severity and frequency of behavior)[6]. For example, studies showed that dual harmers engaged in more frequent and wider range of harmful behaviors, with increased use of lethal methods[7]. 
In view of the high-risk behavior pattern of the dual-harm population, further studies in this field are necessary.

Individuals engaging in dual-harm are over-represented in forensic and clinical population[7, 8], despite its relatively low incidence in the general population[1]. A study of 326 imprisoned individuals reported that about $42 \%$ of the participants had engaged in both aggression and self-harm during their incarceration[3]. Notably, participants in this study were mostly violent offenders, indicating that violent offenders are a high-risk group for dual-harm. However, more attention has been given to these individuals' aggression towards others instead of harm to themselves[4]. When faced with a violent offender, the usual focus of response is to protect others with the use of punishment, containment or seclusion. However, the punishment-oriented strategies are more likely to increase the risk of future self-harm and antisocial behaviors[9]. Therefore, sufficient awareness of the duality of self-harm and aggression is critical for the successful intervention in violent offenders.

To date, risk factors of dual-harm still remained unclear. Efforts have been put into the investigations on associated factors for dual-harm in the last decade, with special attention to childhood adversities (CAs). Some studies, by distinguishing dual harmers from those who engaged only in self-harm or violence towards others, found that childhood adverse experiences might be an independent risk factor for dualharm[10,11]. However, previous studies used many different indicators of CAs, such as childhood maltreatment[2], parental death[12], and low family income[13], and it is still unclear which type of CAs, or CAs as a whole, is associated with dual-harm. Another focus of studies on risk factors of dual-harm is mental disorders. Studies have found that many mental disorders are associated with dual-harm, including substance-related disorders, mood disorders, and posttraumatic stress disorder. For instance, Richmond et al. found that dual harmers showed higher comorbidity of substance dependence than single harmers[2]. Steeg et al. found that approximately two thirds of individuals who dual harm were diagnosed with substance use disorders[5]. Harford et al. found that dual-harm was closely linked to alcohol/drug use disorder, mood disorders and posttraumatic stress disorder[14]. Of note, previous studies have mainly focused on substance-related disorders, while relatively few studies investigated the relationship between dual-harm and non-substance-related mental disorders.

The relationship between personality and self-harm or aggression has been well-established, while its relationship with dual-harm still remains unclear. A previous study revealed that dual harmers were more likely to exhibit personality traits related to emotional and interpersonal liability[2]. Psychopathy is a personality construct marked by interpersonal, affective, and behavioral dysfunction combined with a tendency to express antisocial behaviors[15]. Multiple studies revealed that higher levels of psychopathy was associated with a higher level of delinquent behaviors[16, 17]. Recently, some studies supported that psychopathy, particularly lifestyle-antisocial factors, was associated with suicide-related behaviors [1821]. Based on the above findings, we speculated that psychopathy might be a potential risk factor of dual-harm. 
The concept of dual-harm was proposed in the last few years, and our knowledge about it remains limited. As psychiatric assessments are not routinely performed in violent offenders due to the separation of mental health and justice systems, our knowledge is even more limited about the factors for dual-harm in violent offenders. Based on existing empirical studies, we hypothesized that dual-harm offenders might be impacted by different factors, as compared to those who engaged in violence towards self or others only; and the factors for dual-harm might include childhood adversities, history of mental disorders, substance abuse, psychiatric symptoms, and psychopathy. In this case-control study, we aimed to identify associated factors for dual-harm among violent offenders.

\section{Methods}

\section{Study setting and participants}

This study is a multi-center, case-control study started in May 2013 and ended in January 2016. Data were obtained from seven qualified forensic institutes located in seven different provinces in China. Targeted participants were individuals with violent behaviors who were suspected to have mental disorders. In China, individuals who are engaged in violent behaviors and suspected to have a mental disorder are required to undergo a forensic psychiatric assessment in order to ascertain their mental status. More details about forensic psychiatric assessment in China can be found in previous literature[22]. The inclusion criteria were individuals who (1) were either female or male, aged over 14, (2) perpetrated any violent criminal acts, including homicide, assault, robbery, arson, and sexual offences, (3) were able to understand and answer the questions in the interview, and (4) were willing to participant in this study. Individuals with incomplete records of the information needed in this study were excluded. The study protocol was approved by the Ethics Committee of the Second Xiangya Hospital. All methods were performed in accordance with relevant guidelines and regulations. Informed consent was obtained from all subjects.

\section{Definition of dual-harm}

Dual-harm was defined as the co-occurrence of self-harm and aggression during an individual's lifetime. In this study, we defined self-harm as any self-directed injurious behavior with suicidal intent but not resulting in death (i.e., suicide attempt or self-directed injurious behaviors, including poisoning, use of asphyxiating gas, use of sharp objects, hanging, jumping from height, and drowning). Aggression in this study was defined as any violent criminal acts towards others, including homicide, assault, robbery, arson, and sexual offences.

\section{Instruments and evaluation}

Lifetime history of self-harm was obtained using a self-report questionnaire, which included questions such as "Have you ever made a suicide attempt, for example, cutting yourself or overdosing on drugs?" 
Violent behaviors were ascertained from the participants' forensic archives in this study.

Demographic and clinical information was obtained using a standardized data collection form. The demographic information included sex, age, employment status, marital status, place of residence and education levels, and the clinical information included history of mental disorders, family history of mental disorders, history of head trauma, history of alcohol and non-alcohol substance abuse, previous violence behaviors, and lifetime treatment for mental disorders; all the above data were obtained from the participants' forensic archives.

Each participant was evaluated by at least two senior psychiatrists with the use of the International Classification of Diseases 10 (ICD-10). Diagnoses of mental disorders were made for participants who meet the corresponding diagnostic criteria.

A clinician-rated scale was used to assess the participants' childhood adverse experiences. This scale was designed by our research team based on previous studies of violence risks associated with the circumstances in China. This scale is used to assess the adverse experiences before the age of 15 years, including child abuse, parental abandonment, parental death, parental separation, parental psychiatric disorder, parental criminal behaviors and parental alcohol/non-alcohol substance abuse. Information regarding childhood adverse experiences was obtained from participants by asking questions such as "During the first 15 years of your life, have you ever been abused by your parents?" and "Have you lost any of your parents because he/she died? " The total number of childhood adversities that the participant had experienced were recorded and used in subsequent statistical analyses.

The Chinese version of Psychopathy checklist-Revised (PCL-R) was used to assess psychopathic personality traits in this study[23]. The 20-item PCL-R covered four factors of psychopathy, i.e., interpersonal, affective, lifestyle, and antisocial factors; each item was rated on a 3-point scale (0-2), with the total score ranged $0-40$. For this scale, higher scores indicated a higher degree of psychopathy, with a cutoff score of 25 suggesting the presence of psychopathy. This scale has demonstrated satisfactory reliability and validity in previous studies[24].

The 20-item Chinese version of Brief Psychiatric Rating Scale (BPRS) was used to assess psychiatric symptoms in the participants. The BPRS is a well-established clinician-rated tool consisting of 5 subscales: affect (e.g., anxiety, depression), negative symptoms (e.g., withdrawal), positive symptoms (e.g., thought disturbance), activation (e.g., excitement), and resistance (e.g., hostility, suspiciousness). Each item was rated from 1 (no symptom) to 7(extremely severe), and the score of each subscale was calculated by computing the arithmetic mean of the scores of all items included in the subscale.

\section{Statistical analysis}

Categorical variables were presented as number (percentage), and continuous variables were presented as mean \pm standard deviation. The participants' education level was categorized into high (years of 
education >12), intermediate (years of education ranged 6-12), or low (illiterate or years of education $<6$ ) levels. The diagnoses were classified into five categories: no mental disorder, schizophrenia spectrum disorders, mood disorders, substance-related disorders and other mental disorders (including personality disorders, neurotic disorders, mental retardation, and other non-specified mental disorders, given the small number of cases). Categorical variables were analyzed using Chi-square test, and Fisher's exact test was applied as appropriate. The one-sample Kolmogorov-Smirnov test was used to assess the normality of continuous data. Normally distributed data were analyzed using two-simple t-test and nonnormally distributed data were analyzed using the Mann-Whitney $U$ test. Binary logistic regression analysis was performed to investigate the association between dual-harm and other factors, with all the categorical variables set as dummy variables. The univariate analysis was first performed to screen potential associated factors, and then the forward stepwise (Likelihood Ratio method) procedures were used to identify independent associated factors for dual-harm. With the type of harm (dual-harm or violence-only) set as the dependent variable, the factors with $p<0.1$ in the univariate analysis were selected for the multivariable analysis. All analyses were two-sided, and $p<0.05$ were considered as statistically significant. All data were analyzed using the SPSS software (Version 24.0; IBM).

\section{Results}

\section{Demographic characteristics}

A total of 515 participants were recruited during the study period. Of them, 82 participants were excluded for not meeting the criteria for violent crime and 4 were excluded for incomplete information. The final sample included 429 participants, with $74(17.2 \%)$ participants with self-harm history assigned into the dual-harm (D-H) group and 355 (82.8\%) participants without self-harm history assigned into the violenceonly (V-O) group. A significant difference was found in gender distribution between the two groups ( $\left.x^{2}=4.59, d f=1, p=0.048\right)$, and no significant differences were found in other demographic factors, as shown in Table 1.

\section{Clinical characteristics and childhood adverse experiences}

Compared to the V-O group, participants in the $\mathrm{D}-\mathrm{H}$ group were more likely to have a history of mental disorders $(68.9 \%$ vs $55.5 \%, p<0.05)$. There was no significant difference in other clinical characteristics between the two groups. Among the seven indicators of CAs (see Method), the most prevalent childhood adverse events overall were child abuse (9.3\%) and parental separation (9.1\%). The D-H group exhibited a higher level of exposure to child abuse and parental death during their childhood, as compared to the V-O group $(17.6 \%$ and $14.9 \%$ vs $7.6 \%$ and $4.5 \%, p<0.05)$. No significant inter-group difference was found in the other indicators and the total number of CAs. (Table 2)

\section{Current psychiatric diagnoses}


Overall, there were 359 (83.7\%) participants diagnosed with mental disorders, with the most common diagnoses being schizophrenia spectrum disorders, followed by mood disorders and substance-related disorders. The proportion of participants diagnosed with mood disorders and substance-related disorders was higher in the $\mathrm{D}-\mathrm{H}$ group than in the $\mathrm{V}-\mathrm{O}$ group ( $20.3 \%$ and $12.2 \%$ vs $6.5 \%$ and $7.6 \%)$, and the proportion of participants diagnosed with schizophrenia spectrum disorders and other mental disorders were lower in the $\mathrm{D}-\mathrm{H}$ group than in the $\mathrm{V}-\mathrm{O}$ group ( $48.6 \%$ and $4.1 \%$ vs $57.7 \%$ and $11.5 \%$ ). Compared to those in the V-O group, participants in the $\mathrm{D}-\mathrm{H}$ group had a higher score of the total PCL-R $(\mathrm{T}=-2.06$, $\mathrm{p}=0.039)$ and the anti-social subscale $(\mathrm{T}=-2.21, \mathrm{p}=0.03)$, as well as a higher score of the affective subscale in BPRS $(\mathrm{T}=-3.46, \mathrm{p}=0.001)$. (Table 3$)$

\section{Associated factors for dual-harm}

Current diagnoses of mood disorders (OR=3.03, 95\%Cl: 1.15 - 7.97), child abuse (OR=2.92, 95\%Cl: 1.33 $6.40)$, parental death $(\mathrm{OR}=2.79,95 \% \mathrm{Cl}: 1.14-6.81)$, and the score of the affective subscale in $B P R S(\mathrm{OR}=1.77,95 \% \mathrm{Cl}: 1.31-2.39)$ were independently associated with increased risk of dual-harm in violent offenders. The results are presented in Figure 1.

\section{Discussion}

To our knowledge, this is the first study to investigate the independent factors for dual-harm among violent offenders in China. In the present study, we used a case-control study design and found that current diagnoses of mood disorders, child abuse, parental death and the score of the affective subscale in BPRS were significantly associated with dual-harm among violent offenders.

Childhood adversities may contribute to the development of many maladaptive behaviors, including aggression, self-harm, and violence[25, 26]. Our findings suggested that dual harmers had higher levels of exposure to child abuse and parental death during their childhood, as compared with the individuals engaging in violence towards others only. This was consistent with previously reported findings; for example, Richmond et al. found that dual harmers exhibited more experience of childhood maltreatment than those engaging in violence towards others[2]. A prior study found that parental death was associated with increased risk of both self-harm and violent crime[12], and some researchers found that the risk of dual-harm among individuals who experienced parental death was even greater[10]. However, inconsistent with previous studies, we did not find the association between the total number of CAs and dual-harm. A longitudinal cohort study, which used nearly the same indicators of CAs as those in our study, revealed that individuals with a history of violent offenses who had also experienced cumulative childhood adversities had a higher risk of suicide[11]. Another study using different indicators of CAs found that the experience of five or more childhood adverse events was more frequently observed in dual harmers[10]. The above findings suggested that the type of CAs, instead of the number of CAs, was associated with dual-harm. This was not unexpected, since child abuse was believed to be associated with long-term emotional and physical health problems[27], and parental death was regarded as the most 
stressful event during an individual's upbringing and may lead to lasting adverse consequences[28]. However, as many other indicators of CAs were not included in the present study, future studies are needed to verify the above findings.

The relationship between non-substance-related mental disorders and dual-harm has not been adequately studied. Our study found that dual harmers were more likely to be diagnosed with mood disorders compared to those who only engaged in violence towards others, which was in consistent with the previous findings. Harford et al. reported that dual-harm was more strongly associated with mood disorders[14]. Furthermore, it has been reported that depression is the most common diagnosis in individuals who engaged in murder-suicide, an extreme dual-harm behavior[29]. The above results suggested that individuals with mood disorders needed special attention in studies on dual-harm. Other mental disorders should not be ignored either. A previous study showed that dual harmer had higher rates of psychiatric comorbidity and were faced with more difficulties, but they might not be able to receive additional mental health services[2], indicating that more attention should be given to the comorbidity of mental disorders in dual-harm individuals. Nevertheless, we did not find the association between dualharm and substance-related disorders (including history of substance abuse and current diagnoses of substance-related disorders), which was considered to be a major risk factor for dual-harm in western countries[14, 30,31]. It should be noted that the patterns of substance abuse differed greatly between different regions[32, 33]. For example, studies in Asian countries reported lower rates of comorbidity of substance abuse among individuals with mental disorders, as compared with those in western countries[33]. Thus, the relationship between substance-related disorders and dual-harm in China still needs further research.

Another finding in the present study was that the dual-harm group scored higher on the affective subscale in BPRS than the violence-only group, and this difference persisted after accounting for known risk factors. Few studies have focused on psychiatric symptoms in dual harmers, and only one study reported that individuals with dual-harm ideation had higher scores related to depression than those who had violence ideation only[34]. This result could be explained from the following perspectives. From the psychodynamic aspect, individuals with depression tend to use the defense mechanism of introjection, leading to aggression towards themselves[35]. In addition, some empirical studies revealed that anxiety and depressive symptoms were strongly related to self-oriented violence, rather than violence towards others[36, 37]. Finally, according to the theories about dual-harm, depression might lead to aggression towards self[38].

Some researchers proposed that a personality characterized by a predisposition to engage in harmful behaviors might be shaped by biological factors interacting with adverse early environments [39]; they emphasized that the secondary psychopathy characterized by antisocial and unstable lifestyle could be a risk factor for dual-harm[39]. In our study, the dual-harm group scored higher on the antisocial scale than the violence-only group, which supported the previous views. However, this variable was not entered into the final regression model. Given some empirical evidence supporting psychopathy as a key factor for self-harm and aggression[19, 40], the role of psychopathy in dual-harm cannot be neglected. The 
present results could be attributed to the methodology, since some studies revealed a significant association between psychopathy and self-harm when self-rated scales were used[41]. Therefore, methods for the measurement of psychopathy need to be carefully selected in future studies.

Despite the strength of our study, some limitations should also be noted. First, participants in our study were individuals with violent behaviors who underwent forensic psychiatric assessments, thus, the extrapolation of our findings to other populations needs further validation. Second, the definition of selfharm in this study was limited to suicide attempt, whereas aggression was limited to violent criminal behaviors; the relatively narrow scope of the definitions might have led to underestimation of the rate of dual-harm in the selected population. Third, a self-reported scale was used to obtain self-harm information from the participants, which might lead to memory biases. Fourth, due to the retrospective nature of this study, some important factors were not included.

\section{Conclusions}

Although some factors for dual-harm have been identified, this phenomenon has not been fully understood. In the present study, we investigated several factors known to be associated with dual-harm, such as childhood adversities, substance abuse, and history of mental disorders, and explored some potentially associated factors, such as psychopathology and psychopathy. We found that factors associated with dual-harm among violent offenders in China are different, to some extent, from those observed in other countries. Thus, a comprehensive assessment and detailed evaluation is needed for violent offenders, with highlights of factors associated with self-harm, especially childhood adverse events and psychiatric symptoms. The identified risk factors for dual-harm could provide an insight into the development of effective intervention strategies for violent offenders.

\section{Abbreviations}

CAs: childhood adversities.

D-H: dual-harm

V-O: violence-only

\section{Declarations}

\section{Ethics approval and consent to participate}

The study protocol was approved by the Human Ethics Committee of the Second Xiangya Hospital of Central South University. All methods were performed in accordance with the study protocol and ethical guidelines and regulations. Signed written informed consent was obtained from all subjects. 


\section{Consent to publish}

Not applicable.

\section{Availability of data and materials}

The data generated or analyzed during this study are included in this manuscript and its Supplementary materials.

\section{Competing interests}

The authors claim that there are no conflicts of interest.

\section{Funding}

This work was supported by the Fundamental Research Funds for the Central Universities of Central South University(2021zzts0395), National Natural Science Foundation of China (Grant number: 82071543), Science and Technology Program of Hunan Province (2018SK2133), Hunan Innovative Province Construction Project (2019SK2334) and Natural Science Foundation of Hunan Province (2019JJ40424). The funder had no role in study design, data collection, interpretation, data analysis, and paper writing.

\section{Authors' contributions}

YH conceived the study, performed the date analysis and drafted the manuscript. XW and JZ were responsible for interpretation of the data and revising the manuscript. Simei Zhang, NG, and QS were involved in the acquisition of the data. Shaoling Zhong, HG, RL, WG, HC, JW were engaged in the analysis for the work. All authors have read and agreed with the final manuscript.

\section{Acknowledgment}

We would like to thank all participants for their contribution to this study. We also thank the following institutes: West China Hospital, Sichuan University, Affiliated Brain Hospital of Guangzhou Medical University, Beijing Ankang Hospital, Xiamen Xianyue Hospital, Nanjing Brain Hospital, and Guangxi Prison General Hospital for their support.

\section{References}


1. Sahlin H, Kuja-Halkola R, Bjureberg J, Lichtenstein P, Molero Y, Rydell M, Hedman E, Runeson B, Jokinen J, Ljótsson Bet al.Association Between Deliberate Self-harm and Violent Criminality. JAMA Psychiatry2017, 74(6):615-621. doi:10.1001/jamapsychiatry.2017.0338

2. Richmond-Rakerd LS, Caspi A, Arseneault L, Baldwin JR, Danese A, Houts RM, Matthews T, Wertz J, Moffitt TE.Adolescents Who Self-Harm and Commit Violent Crime: Testing Early-Life Predictors of Dual Harm in a Longitudinal Cohort Study. Am J Psychiatry2019, 176(3):186195.doi:10.1176/appi.ajp.2018.18060740

3. Slade K.Dual harm: an exploration of the presence and characteristics for dual violence and selfharm behaviour in prison. Journal of Criminal Psychology2018, 8(2):97-111.doi:10.1108/jcp-032017-0017

4. Slade K.Dual harm: the importance of recognising the duality of self-harm and violence in forensic populations. Med Sci Law2019, 59(2):75-77.doi:10.1177/0025802419845161

5. Steeg S, Webb RT, Mok PLH, Pedersen CB, Antonsen S, Kapur N, Carr MJ.Risk of dying unnaturally among people aged 15-35 years who have harmed themselves and inflicted violence on others: a national nested case-control study. Lancet Public Health2019, 4(5):e220-e228.doi:10.1016/s24682667(19)30042-8

6. O'Donnell O, House A, Waterman M.The co-occurrence of aggression and self-harm: systematic literature review. J Affect Disord2015, 175:325-350.doi:10.1016/j.jad.2014.12.051

7. Slade K, Forrester A, Baguley T.Coexisting violence and self-harm: Dual harm in an early-stage male prison population. Legal and Criminological Psychology2020, 25(2):182-198.doi:10.1111/lcrp.12169

8. Nijman HL, JM àC.Situational determinants of inpatient self-harm. Suicide Life Threat Behav2002, 32(2):167-175.doi:10.1521/suli.32.2.167.24401

9. Kaba F, Lewis A, Glowa-Kollisch S, Hadler J, Lee D, Alper H, Selling D, MacDonald R, Solimo A, Parsons Aet al.Solitary confinement and risk of self-harm among jail inmates. Am J Public Health2014, 104(3):442-447.doi:10.2105/ajph.2013.301742

10. Carr MJ, Steeg S, Mok PLH, Pedersen CB, Antonsen S, Kapur N, Webb RT.Adverse Childhood Experiences and Risk of Subsequently Engaging in Self-Harm and Violence towards Other People"Dual Harm". Int J Environ Res Public Health2020, 17(24).doi:10.3390/ijerph17249409

11. Björkenstam E, Hjern A, Björkenstam C, Kosidou K.Association of Cumulative Childhood Adversity and Adolescent Violent Offending With Suicide in Early Adulthood. JAMA Psychiatry2018, 75(2):185193.doi:10.1001/jamapsychiatry.2017.3788

12. Carr MJ, Mok PLH, Antonsen S, Pedersen CB, Webb RT.Self-harm and violent criminality linked with parental death during childhood. Psychol Med2020, 50(7):1224-

1232.doi:10.1017/s0033291719001193

13. Mok PLH, Antonsen S, Pedersen CB, Carr MJ, Kapur N, Nazroo J, Webb RT.Family income inequalities and trajectories through childhood and self-harm and violence in young adults: a population-based, nested case-control study. Lancet Public Health2018, 3(10):e498-e507.doi:10.1016/s24682667(18)30164-6 
14. Harford TC, Chen CM, Kerridge BT, Grant BF.Self- and other-directed forms of violence and their relationship with lifetime DSM-5 psychiatric disorders: Results from the National Epidemiologic Survey on Alcohol Related Conditions-III (NESARC-III). Psychiatry Res2018, 262:384392.doi:10.1016/j.psychres.2017.09.012

15. Walsh Z, Swogger MT, Walsh T, Kosson DS.Psychopathy and violence: increasing specificity. Neth J Psycho/2007, 63(4):125.doi:10.1007/bf03061075

16. McCuish EC, Corrado RR, Hart SD, DeLisi M.The role of symptoms of psychopathy in persistent violence over the criminal career into full adulthood. Journal of Criminal Justice2015, 43(4):345356.doi:10.1016/j.jcrimjus.2015.04.008

17. Gray NS, Snowden RJ.Psychopathy in women: Prediction of criminality and violence in UK and USA psychiatric patients resident in the community. Psychiatry Res2016, 237:339-

343.doi:10.1016/j.psychres.2016.01.014

18. Pennington CR, Cramer RJ, Miller HA, Anastasi JS.Psychopathy, depression, and anxiety as predictors of suicidal ideation in offenders. Death Stud2015, 39(1-5):288-

295.doi:10.1080/07481187.2014.991953

19. Heirigs MH, DeLisi M, Fox B, Dhingra K, Vaughn MG.Psychopathy and Suicidal Thoughts and Behaviors Revisited: Results From a Statewide Population of Institutionalized Youth. Int $\mathrm{J}$ Offender Ther Comp Crimino/2019, 63(6):874-895.doi:10.1177/0306624×18812533

20. Verona E, Sprague J, Javdani S.Gender and factor-level interactions in psychopathy: implications for self-directed violence risk and borderline personality disorder symptoms. Personal Disord2012, 3(3):247-262.doi:10.1037/a0025945

21. Smith PN, Selwyn CN, Wolford-Clevenger C, Mandracchia JT.PSYCHOPATHIC PERSONALITY TRAITS, SUICIDE IDEATION, AND SUICIDE ATTEMPTS IN MALE PRISON INMATES. Crim Justice Behav2014, 41(3):364-379.doi:10.1177/0093854813506884

22. Li G, Gutheil TG, Hu Z.Comparative study of forensic psychiatric system between China and America. Int J Law Psychiatry2016, 47:164-170.doi:10.1016/j.ijlp.2016.04.002

23. Hare RD, Neumann CS.Structural models of psychopathy. Curr Psychiatry Rep2005, 7(1):5764.doi:10.1007/s11920-005-0026-3

24. Hare RD, Clark D, Grann M, Thornton D.Psychopathy and the predictive validity of the PCL-R: an international perspective. Behav Sci Law2000, 18(5):623-645.doi:10.1002/10990798(200010)18:5<623::aid-bs|409>3.0.co;2-w

25. Fox BH, Perez N, Cass E, Baglivio MT, Epps N.Trauma changes everything: examining the relationship between adverse childhood experiences and serious, violent and chronic juvenile offenders. Child Abuse Neg/2015, 46:163-173.doi:10.1016/j.chiabu.2015.01.011

26. Brent DA, Melhem NM, Oquendo M, Burke A, Birmaher B, Stanley B, Biernesser C, Keilp J, Kolko D, Ellis Set al.Familial pathways to early-onset suicide attempt: a 5.6-year prospective study. JAMA Psychiatry2015, 72(2):160-168.doi:10.1001/jamapsychiatry.2014.2141 
27. Gilbert R, Widom CS, Browne K, Fergusson D, Webb E, Janson S.Burden and consequences of child maltreatment in high-income countries. Lancet2009, 373(9657):68-81.doi:10.1016/s01406736(08)61706-7

28. Melhem NM, Porta G, Shamseddeen W, Walker Payne M, Brent DA.Grief in children and adolescents bereaved by sudden parental death. Arch Gen Psychiatry2011, 68(9):911919.doi:10.1001/archgenpsychiatry.2011.101

29. Marzuk PM, Tardiff K, Hirsch CS.The epidemiology of murder-suicide. Jama1992, 267(23):31793183.doi: 10.1001/jama.1992.03480230071031

30. Harford TC, Yi HY, Grant BF.Other- and self-directed forms of violence and their relationships to DSMIV substance use and other psychiatric disorders in a national survey of adults. Compr Psychiatry2013, 54(7):731-739.doi:10.1016/j.comppsych.2013.02.003

31. Kinner SA, Borschmann R.Dual-harm, complex needs, and the challenges of multisectoral service coordination. Lancet Public Health2019, 4(5):e210-e211.doi:10.1016/s2468-2667(19)30065-9

32. Cheng HG, Phillips MR, Li X, Zhang J, Shi Q, Xu G, Song Z, Ding Z, Pang S.Co-occurrence of DSM-IV mental disorders and alcohol use disorder among adult Chinese males. Psychol Med2017, 47(16):2811-2822.doi:10.1017/s0033291717001337

33. Thirthalli J, Kumar CN, Arunachal G.Epidemiology of comorbid substance use and psychiatric disorders in Asia. Curr Opin Psychiatry2012, 25(3):172-180.doi:10.1097/YC0.0b013e3283523c26

34. Hemming L, Shaw J, Haddock G, Carter LA, Pratt D.A Cross-Sectional Study Investigating the Relationship Between Alexithymia and Suicide, Violence, and Dual Harm in Male Prisoners. Front Psychiatry2021, 12:670863.doi:10.3389/fpsyt.2021.670863

35. Boeker H, Kraehenmann R.Neuropsychodynamic Approach to Depression: Integrating Resting State Dysfunctions of the Brain and Disturbed Self-Related Processes. Front Hum Neurosci2018, 12:247.doi:10.3389/fnhum.2018.00247

36. Melhem NM, Porta G, Oquendo MA, Zelazny J, Keilp JG, lyengar S, Burke A, Birmaher B, Stanley B, Mann JJet al.Severity and Variability of Depression Symptoms Predicting Suicide Attempt in HighRisk Individuals. JAMA Psychiatry2019, 76(6):603-613.doi:10.1001/jamapsychiatry.2018.4513

37. Beck AT, Steer RA, Beck JS, Newman CF.Hopelessness, depression, suicidal ideation, and clinical diagnosis of depression. Suicide Life Threat Behav1993, 23(2):139-145.doi:

38. Plutchik R.Outward and inward directed aggressiveness: the interaction between violence and suicidality. Pharmacopsychiatry1995, 28 Suppl 2:47-57.doi:10.1055/s-2007-979620

39. Shafti M, Taylor PJ, Forrester A, Pratt D.The Co-occurrence of Self-Harm and Aggression: A CognitiveEmotional Model of Dual-Harm. Front Psycho/2021, 12:586135.doi:10.3389/fpsyg.2021.586135

40. Hare RD.Psychopathy as a risk factor for violence. Psychiatr Q1999, 70(3):181197.doi:10.1023/a:1022094925150

41. Douglas KS, Herbozo S, Poythress NG, Belfrage H, Edens JF.Psychopathy and suicide: A multisample investigation. Psychological Services2006, 3(2):97-116.doi: 10.1037/1541-1559.3.2.97 


\section{Tables}

Table 1

Demographic characteristics of the D-H and V-O groups

\begin{tabular}{|c|c|c|c|c|c|}
\hline \multirow[t]{2}{*}{ Variable } & \multirow[t]{2}{*}{ Total (429) } & & & & \\
\hline & & D-H (74) & V-O (355) & Statistics & $P$ \\
\hline Sex & & & & $4.588^{a}$ & 0.048 \\
\hline Male & $388(90.4)$ & $62(83.8)$ & $326(91.8)$ & & \\
\hline Female & $41(9.6)$ & $12(16.2)$ & $29(8.2)$ & & \\
\hline \multicolumn{6}{|l|}{ Age } \\
\hline Mean $\pm S D$ & & $33.53 \pm 9.29$ & $34.15 \pm 10.12$ & $0.490^{b}$ & 0.624 \\
\hline Unemployment & $223(52.0)$ & $33(44.6)$ & $190(53.5)$ & $1.955^{\mathrm{a}}$ & 0.201 \\
\hline Marital status & & & & $1.373^{c}$ & 0.727 \\
\hline Never married & 239 (55.7) & $44(59.5)$ & 195 (54.9) & & \\
\hline Married & $121(28.2)$ & $17(23.0)$ & 104 (29.3) & & \\
\hline Divorced & $58(13.5)$ & $11(14.9)$ & $47(13.2)$ & & \\
\hline Widowed & $11(2.6)$ & $2(2.7)$ & $9(2.5)$ & & \\
\hline Residence & & & & $1.648^{a}$ & 0.204 \\
\hline Urban & 203 (47.3) & $30(40.5)$ & $173(48.7)$ & & \\
\hline Rural & $226(52.7)$ & $44(59.5)$ & $182(51.3)$ & & \\
\hline Education ${ }^{1}$ & & & & $0.72^{\mathrm{C}}$ & 0.71 \\
\hline Low & 282 (65.7) & $52(70.3)$ & $230(64.8)$ & & \\
\hline Medium & $127(29.6)$ & 19(25.7) & $108(30.4)$ & & \\
\hline High & $20(4.7)$ & $3(4.1)$ & $17(4.8)$ & & \\
\hline
\end{tabular}

Education $^{1}$ : low: $\leq 6$ years of education, medium: $6-12$ years of education, high: $>12$ years of education. ${ }^{\text {a. }}$ Chi-squared test, ${ }^{\text {b. }}$ Two samples t test, ${ }^{\text {c. }}$ Fisher's exact test.

\section{Table 2}

Clinical characteristics and childhood adverse experiences of the D-H and V-O groups 
Variable

Total (429)

$\begin{array}{lll}\text { D-H (74) } & \text { V-O } \\ & (355)\end{array} \quad$ Statistics $\quad P$

History of mental disorders, $\mathrm{n}(\%)$

$248(57.8)$

51

(68.9)

197

(55.5)

$4.526^{\mathrm{a}}$

0.038

Family history of mental disorders, $\mathrm{n}(\%) \quad 69(16.1)$

$\begin{array}{llll}16 & 53 & 2.032^{\mathrm{a}} & 0.165 \\ (21.6) & (14.9) & & \end{array}$

History of head trauma, n (\%)

$42(9.8)$

12(16.2) $30(8.5)$

$4.181^{\mathrm{a}}$

0.052

History of alcohol abuse, $\mathrm{n}(\%)$

67 (15.6) $\quad 8(10.8)$

59

(16.6)

$1.568^{\mathrm{a}}$

0.225

History of non-alcohol substance

abuse, $\mathrm{n}(\%)$

$48(11.2)$

13

(17.6)

35 (9.9)

$3.662^{\mathrm{a}}$

0.067

Previous violence history, $\mathrm{n}(\%)$

$375(87.4)$

66

(89.2)

309

(87.0)

$0.257^{a}$

0.703

Treatment experience

$1.728^{\mathrm{c}}$

0.430

Untreated

Outpatient

Inpatient

Indicators of childhood adversity

Child abuse, $n(\%)$

Parental abandonment, $\mathrm{n}(\%)$

Parental death

$$
40 \text { (9.3) }
$$

13

(17.6)

$15(3.5)$

$3(4.1)$

$27(6.3)$

11
$(14.9)$
139

(39.2)

71

145

(40.8)
(25.7) (20.0)

$\begin{array}{ll}31 & 145 \\ (41.9) & (40.8)\end{array}$




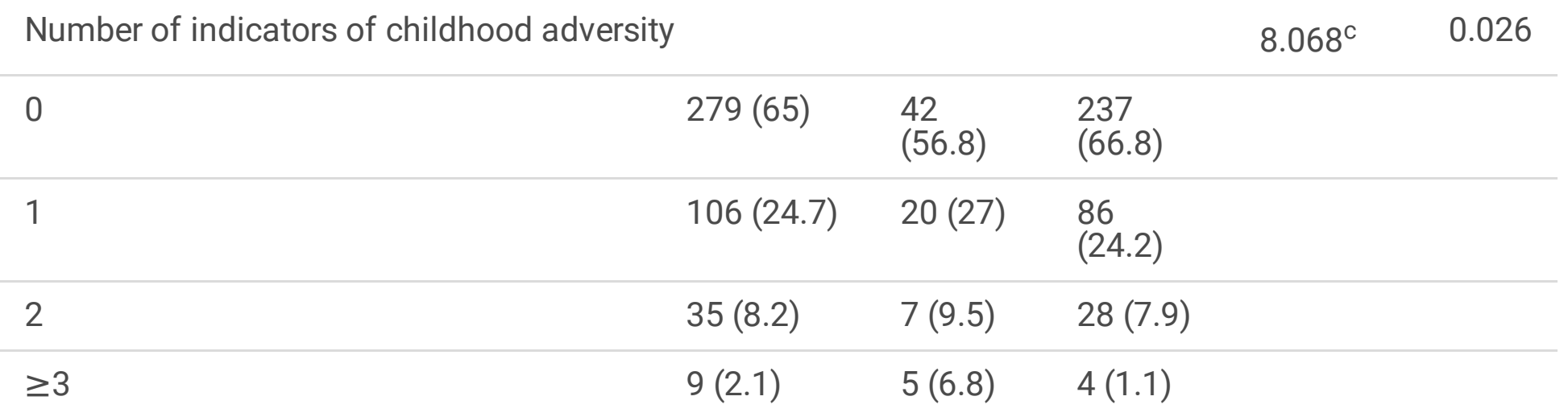

a: Chi-squared test, ${ }^{c}$ : Fisher's exact test.

\section{Table 3}

Current psychiatric diagnoses and assessments in the $\mathrm{D}-\mathrm{H}$ and $\mathrm{V}-\mathrm{O}$ groups 


\begin{tabular}{|c|c|c|c|c|c|}
\hline \multirow[t]{2}{*}{ Variable } & \multirow[t]{2}{*}{ Total (429) } & & & & \\
\hline & & $\mathrm{DH}(74)$ & VO (355) & Statistics & $P$ \\
\hline Current diagnoses & & & & $16.943^{c}$ & 0.002 \\
\hline Non-psychiatry & $70(16.3 \rrbracket$ & $11(14.9 \rrbracket$ & $59(16.6)$ & & \\
\hline $\begin{array}{l}\text { Schizophrenia spectrum } \\
\text { disorders }\end{array}$ & $241(56.2)$ & $36(48.6)$ & $205(57.7)$ & & \\
\hline Mood disorders & $38(8.9)$ & $15(20.3)$ & $23(6.5)$ & & \\
\hline Substance-related disorders & $36(8.4)$ & $9(12.2)$ & $27(7.6)$ & & \\
\hline Other & $44(10.3)$ & $3(4.1)$ & $41(11.5)$ & & \\
\hline \multicolumn{6}{|l|}{ PCL-R } \\
\hline Total & & $14.38 \pm 9.82$ & $12.16 \pm 8.08$ & $-2.06^{b}$ & 0.039 \\
\hline Interpersonal & & $1.73 \pm 2.98$ & $1.68 \pm 1.94$ & $-0.20^{\mathrm{b}}$ & 0.846 \\
\hline Emotion & & $2.78 \pm 2.25$ & $2.72 \pm 2.51$ & $-0.21^{b}$ & 0.834 \\
\hline Lifestyle & & $5.26 \pm 3.02$ & $4.53 \pm 3.24$ & $-1.77^{\mathrm{b}}$ & 0.078 \\
\hline Anti-social & & $3.55 \pm 2.78$ & $2.80 \pm 2.16$ & $-2.21^{b}$ & 0.030 \\
\hline \multicolumn{6}{|l|}{ BPRS } \\
\hline Anxiety-depression & & $2.16 \pm 0.89$ & $1.79 \pm 0.85$ & $-3.46^{\mathrm{b}}$ & 0.001 \\
\hline Withdrawal & & $2.06 \pm 1.05$ & $2.13 \pm 1.03$ & $0.52^{b}$ & 0.603 \\
\hline Thought disturbance & & $2.48 \pm 1.25$ & $2.62 \pm 1.31$ & $0.81^{b}$ & 0.421 \\
\hline Activation & & $1.41 \pm 0.66$ & $1.38 \pm 0.79$ & $-0.28^{b}$ & 0.770 \\
\hline Hostile suspicion & & $2.17 \pm 1.12$ & $2.28 \pm 1.28$ & $0.78^{b}$ & 0.438 \\
\hline
\end{tabular}

b. Two simple t-test, c: Fisher's exact test.

\section{Figures}




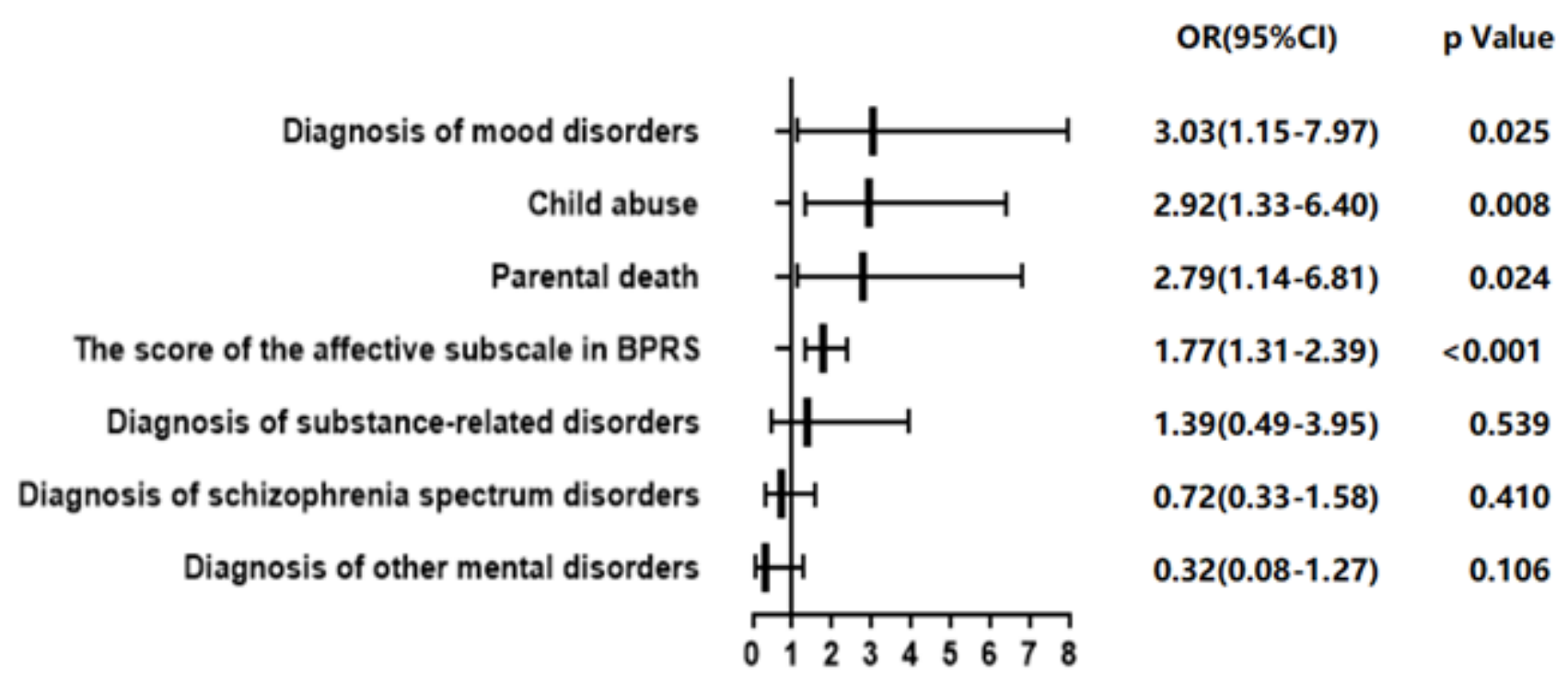

Figure 1

Binary logistic regression analysis of the $\mathrm{D}-\mathrm{H}$ group $(\mathrm{n}=74)$ and the $\mathrm{V}-\mathrm{O}$ group $(\mathrm{n}=355)$

\section{Supplementary Files}

This is a list of supplementary files associated with this preprint. Click to download.

- DataYH.xlsx 